\title{
Essais
}

Revue interdisciplinaire d'Humanités

Résister entre les lignes

Stéphanie Durrans, Moya Jones et Françoise Bonnet, (Se) Construire dans l'interlangue : perspectives transatlantiques sur le multilinguisme

\section{Sarah Dufaure}

\section{(2) OpenEdition \\ Journals}

Édition électronique

URL : http://journals.openedition.org/essais/4816

DOI : 10.4000/essais.4816

ISSN : 2276-0970

Éditeur

École doctorale Montaigne Humanités

Édition imprimée

Date de publication : 15 juillet 2016

Pagination : 198-202

ISBN : 978-2-9544269-8-3

ISSN : 2417-4211

Référence électronique

Sarah Dufaure, «Stéphanie Durrans, Moya Jones et Françoise Bonnet, (Se) Construire dans

l'interlangue : perspectives transatlantiques sur le multilinguisme », Essais [En ligne], 9 | 2016, mis en ligne le 23 octobre 2020, consulté le 29 octobre 2020. URL : http://journals.openedition.org/essais/4816 ; DOI : https://doi.org/10.4000/essais.4816 


\section{Comptes rendus}

\section{Stéphanie Durrans, Moya Jones et Françoise Bonnet (éd.), (Se) Construire}

dans l'interlangue: perspectives transatlantiques sur le multilinguisme, Lille, Septentrion, 2014, $201 \mathrm{p}$.

(Se) Construire dans l'interlangue : perspectives transatlantiques sur le multilinguisme est un ouvrage collectif émanant d'un colloque organisé en mars 2013 à l'Université Bordeaux Montaigne sur les enjeux multiples de l'interlangue et du multilinguisme. Les dix-huit contributions qui composent l'étude convoquent des champs d'étude aussi divers et variés que la littérature, la civilisation ou la didactique, tout en explorant des territoires aussi distincts que le Danemark, le Portugal, la Grèce, l'Italie, l'Algérie, les États-Unis, le Mexique ou l'Irlande. Les actes interrogent ensemble la notion d'interlangue en tant qu'espace intermédiaire labile et dialogique, creuset de la construction identitaire du sujet, de la nation ou du continent tout entier, loin de tout carcan essentialiste. Ils s'appuient sur une définition de l'interlangue en tant que système linguistique et culturel hybride faisant voler en éclats l'acception traditionnelle du multilinguisme comme une simple accumulation ou juxtaposition stérile de monolinguismes. La publication de cet ouvrage semble tout à fait bienvenue dans un contexte mondial où le transfert des idées et la mobilité des personnes ne cessent de s'accélérer, débouchant sur l'émergence d'un multiculturalisme désormais perçu comme une force et non plus une différence à stigmatiser. Les travaux des différents auteurs ont en effet ici pour objectif commun de sonder l'espace de l'interlangue comme un entre-deux générateur de fertilité en matière de construction du sujet là où le multilinguisme, notamment dans le cadre de l'éducation, a souvent été populairement envisagé comme une faiblesse, un inconvénient ou un obstacle, comme un espace interlope, une pierre d'achoppement à l'intégration du sujet et à la pleine maîtrise de la langue institutionnelle promue par le symbole étatique de l'école. 
L'ouvrage s'articule autour de deux parties principales, la première interrogeant la notion de multilinguisme sous un angle essentiellement didactique et la seconde d'un point de vue civilisationniste et littéraire, s'intéressant aux constructions sociales et imaginaires favorisées par l'interlangue. Chacune de ces deux parties se compose de contributions universitaires cherchant à concilier pratique et théorie et se conclue sur deux études de cas venant illustrer de manière encore plus empirique, au moyen de données et de témoignages authentiques récoltés sur le terrain, les problématiques esquissées dans le reste de l'ouvrage. Ces études de terrain portent entre autres sur l'apprentissage des langues étrangères à l'école primaire à travers la lecture d'ouvrages de fiction bilingues pour enfants, l'immersion de groupes d'élèves étrangers dans un environnement à mille lieues de leur langue et de leur culture maternelles, ou encore l'expérience de la traduction collective comme un passage entre deux langues servant de tremplin à la création d'une troisième langue interstitielle, évolutive et dynamique.

Une première partie s'intéresse au microcosme de l'école, comme lieu de rencontres par excellence de langues et de cultures variées. Si l'institution scolaire a pour rôle prépondérant de former les citoyens de demain en leur inculquant un savoir identique et des valeurs communes, elle vise aussi à respecter et promouvoir les parcours individuels de chacun, et à favoriser par là même les échanges interculturels et interlinguistiques pour mieux encourager la tolérance des membres de la communauté éducative, enrichir les enseignements et diversifier la vie en collectivité. Les articles de cette première partie didactique se penchent sur les problématiques courantes d'apprentissage et d'enseignement des langues vivantes dans plusieurs pays. Ils posent tous l'idée que si les contacts entre les langues et les cultures sont le résultat diachronique de mécanismes historiques et géopolitiques, les questions de construction pluri-identitaire qui en découlent restent indissociables d'une réflexion approfondie sur les pratiques pédagogiques du primaire au supérieur. Ils abordent entre autres les difficultés auxquelles l'enseignant de langue étrangère se confronte quand il doit aider ses élèves à construire une identité linguistique et culturelle dans un territoire sur lequel l'ombre du colonialisme plane toujours. Ces articles témoignent de la complexité de la mission confiée aux enseignants de langue dans des contextes pluriels où l'interlangue est le résultat de conflits anciens et où les pratiques d'enseignement doivent composer avec des langues ou des dialectes qui dénotent des statuts politiques particuliers et connotent de fait chez les apprenants certains a priori affectifs.

Les six articles de cette première partie laissent entrevoir sans jamais la nommer explicitement l'épineuse question de la pédagogie différenciée, remettant en cause l'idée d'une évolution homogène de l'interlangue au sein de la classe. Ils partent pour bon nombre du constat que les élèves arrivent à l'école avec une maitrise différente de la langue de scolarité, emportant 
avec eux des vécus spécifiques qui infléchissent de manière inéluctable leur approche et leur acquisition de la langue cible. Bien que les contributions mettent souvent le doigt sur ce problème central de l'hétérogénéité des profils chez les apprenants, elles formulent davantage de questions que de réponses. Par exemple, si Hélène Vasilopoulo analyse l'influence des parcours de jeunes élèves étrangers en Grèce, en particulier des Albanais, sur l'apprentissage des langues, et la difficulté pour les enseignants de s'adapter à ces variations, elle ne fait qu'esquisser des idées de pratiques pédagogiques susceptibles de " filtrer " les spécificités biographiques des apprenants pour les placer dans une situation égale d'apprentissage et de réussite. L'auteur explique que les filtres favorisent l'identification des élèves aux supports pédagogiques tout en maintenant les horizons linguistiques et culturels de chacun pour mieux encourager l'interaction et le transfert des savoirs. Aucun exemple concret de supports pédagogiques pouvant faciliter l'enseignement en classe plurilingue n'est cependant donné, preuve que la richesse et la fertilité incarnées par les langues et les cultures présentes en filigrane dans la classe de langue demandent à faire l'objet d'études plus poussées.

Les articles de cette partie didactique ont néanmoins ceci de fort intéressant qu'ils conçoivent l'école comme un espace favorisant à la fois l'interlangue et la " translangue " ${ }^{1}$, cette dernière pouvant être définie comme un phénomène de va-et-vient constant entre plusieurs langues, comme une nécessaire interpénétration de systèmes linguistiques différents où les langues-cultures s'influencent mutuellement dans une perspective psycholinguistique et sociolinguistique. En reconnaissant le bien-fondé du principe socio-constructiviste (l'idée que l'on apprend essentiellement une langue par comparaison et par confrontation de cette langue avec sa langue maternelle), les contributions de l'ouvrage tordent le cou aux toutes récentes pratiques d'enseignement des langues vivantes dans le primaire et dans le secondaire français qui visent à enjoindre les nouveaux enseignants lauréats des concours à prescrire tout détour par la langue maternelle, pour mieux placer les élèves dans une situation d'immersion totale où seule la langue cible peut et doit être parlée ou écrite.

La seconde partie de l'ouvrage analyse la notion d'interlangue du point de vue de l'Histoire et des politiques nationales linguistiques en lien avec l'immigration en Europe et aux États-Unis. Elle se penche également sur le reflet de ses réalités dans la littérature. Comme le cadre de l'école le laisse entrevoir, l'interlangue représente souvent la réalité quotidienne d'immigrés installés dans un pays exolingue d'accueil. L'introduction de l'ouvrage offre un

1 Sur le concept de translangue, voir Angela Creese \& Adrian Blackledge, "Translanguaging in the Bilingual Classroom: A Pedagogy for Learning and Teaching ", The Modern Language Journal, vol. 94, n 1, 2010, p. 103-115. Voir également Ofelia García \& Li Wei, Translanguaging, Language, Bilingualism and Education, Palgrave Pivot, 2013. 
parcours convaincant des politiques linguistiques de l'Union Européenne qui s'est donnée pour mission ces dernières années de soutenir la diversité linguistique et de promouvoir le multilinguisme au sein de ses frontières, un phénomène qui s'est notamment traduit par l'élaboration du « Programme d'action dans le domaine de l'éducation et de la formation tout au long de la vie ", du Portefeuille européen des langues étrangères (PEL) et du Cadre européen commun de référence pour les langues (CECRL). Un des défis de l'Union Européenne consiste aujourd'hui à offrir au reste du monde un modèle de cohésion idéologique tout en respectant la diversité linguistique et culturelle de ses états membres. L'UE comporte en effet à ce jour 27 États membres tout en reconnaissant 24 langues officielles. L'article de Vanessa Mas-Moury porte ainsi sur la politique de l'Irlande après l'accès à l'indépendance en 1921 et évalue les efforts du gouvernement irlandais à protéger et à promouvoir la langue historique nationale par rapport à l'anglais, à travers la mise en place de cours obligatoires d'irlandais dès l'école primaire.

L'introduction retrace également l'évolution des États-Unis en matière de politiques linguistiques, ou plutôt d'absence de politique linguistique, le pays tendant à favoriser l'hégémonie de l'anglais sur d'autres langues comme l'espagnol. Le désir de conserver ou de promouvoir les langues d'origine des migrants y est malheureusement souvent perçu comme une manifestation d'anti-américanisme. La contribution de James Cohen démontre cependant que les défenseurs de la diversité linguistique sont bien présents aux ÉtatsUnis, démantelant le postulat romantique selon lequel le monolinguisme agit comme le socle d'une identité nationale sédimentaire. James Cohen explore la possibilité de définir un nouveau " modèle de citoyenneté » qui dépasserait les frontières nationales et impliquerait par conséquent une "décolonisation " de la citoyenneté américaine ${ }^{2}$.

Les autres articles de cette seconde partie analysent en outre en quoi les immigrés intègrent la langue et la culture de la terre d'accueil tout en conservant leurs spécificités d'origine pour se construire une nouvelle identité qui contribue peu à peu à décentrer et à déterritorialiser les discours nationaux et les modèles identitaires fossilisés. Le lecteur trouvera dans cette seconde partie un entretien exclusif avec l'écrivaine chicana Carmen Tafolla, récemment visée par la censure aux États-Unis, qui met en lumière sa pratique de l'alternance codique (code-switching) et de l'interlangue dans le plus vaste contexte de l'écriture mexico-américaine. Tafolla y décrit sa conception imagée de la langue comme un arbre dont les multiples branches linguistiques se déploient autour d'un même tronc et y élabore une métaphore de la littérature comme un miroir réfléchissant et réfractant l'identité monolingue, bilingue ou pluri-

2 James Cohen, Spanglish America : Les enjeux de la latinisation des États-Unis, Paris, Le Félin, 2005, p. 24. 
lingue de l'écrivain et du lecteur. Dans le domaine littéraire, le concept d'interlangue s'est consolidé dans les années 1980 pour décrire les phénomènes d'hybridation linguistique au sein d'un même syntagme. Le caractère labile de l'interlangue fascine les universitaires depuis plusieurs décennies, synonyme à la fois de déconstruction et de construction du texte littéraire. Les textes multilingues remettent en cause la pertinence, voire la légitimité, des anciennes littératures dites " nationales ", porteuses d'une seule langue, et tendent à défier et ouvrir le canon dont l'autorité fait désormais polémique tant il ne reflète plus à l'ère moderne la configuration démographique de la culture qu'il est censé refléter. Certains articles, à partir d'une analyse de l'œuvre d'auteurs tels Cormac McCarthy, Olga Gonçalves, Daniel Mendelsohn ou Olive Senior, s'emploient ainsi à interroger la valeur opératoire du concept d'interlangue dans la structuration du discours littéraire, examinant la mise en place de diverses stratégies et dynamiques d'écriture posant la page comme une zone d'interférences linguistiques multiples et foisonnantes.

En fin de compte, quels que soient la méthodologie disciplinaire retenue et le territoire géographique exploré, les auteurs sondent avec efficacité l'influence problématique de l'interlangue sur le processus de construction identitaire dans un monde contemporain en mutation perpétuelle. L'étude exhorte le lecteur à repenser l'identité dans une perspective hétérogène et hybride où la versatilité inhérente aux notions de multilinguisme, d'interlangue et de translangue, garantit l'émergence d'un espace poreux générateur d'une reconfiguration et d'un décentrement constants du sujet.

\author{
Sarah Dufaure \\ EA 4196 CLIMAS \\ Université Bordeaux Montaigne \\ sarah.dufaure@u-bordeaux-montaigne.fr
}

Vittorio Frajese, La censura in Italia. Dall'Inquisizione alla polizia, RomaBari, Laterza, 2014, 240 p.

Il n'existait pas à ce jour une synthèse d'histoire de la censure dans la péninsule italienne à l'époque moderne. Le principal mérite de cet ouvrage est donc de combler cette lacune. Son originalité tient au choix d'une périodisation longue qui correspond au processus que l'auteur identifie comme le fil conducteur de son analyse : la naissance, à la moitié du XVI ${ }^{e}$ siècle, de la censure ecclésiastique ; sa progressive substitution par une police des livres mise en place, dans les différents états italiens, au cours du XVIII et du XIX ${ }^{\mathrm{e}}$ siècle. 